\title{
Innovación educativa a través de tecnologías de la información y comunicación: estudio de caso en un curso de investigación formativa
}

Cardoza-Sernaqué Manuel Antonio, Maestro en gestión del talento humano ${ }^{1}$, Miñan-Olivos Guillermo Segundo, Magíster en gestión pública ${ }^{1}$, Pulido-Joo Luis Alexander, Maestro en gestión del talento humano ${ }^{1}$, Dios-Castillo, Christian Abraham, Magíster

en administración de negocios y Pelaez-Valdivieso José Victor, Doctor en Administración y Manrrique-Luperdi Alexander Miguel, Estudiante de Contabilidad ${ }^{1}$

${ }^{1}$ Universidad Tecnológica del Perú, Perú, mcardoza@utp.edu.pe, c20342@utp.edu.pe, lpulido@utp.edu.pe, cdios@utp.edu.pe, u21202726@utp.edu.pe

${ }^{2}$ Universidad César Vallejo, Perú, mgjosepelaez@gmail.com

\begin{abstract}
This research describes educational innovation through information and communication technologies and proposes a case study in a formative research course where the Covid-19 pandemic led to the need to rethink the entire teaching-learning process. The research was of the non-experimental type with a mixed approach (qualitative and quantitative) and a descriptive scope. The analyzed course was taught in person and at the beginning of the pandemic it became a virtual course where the class was developed through Zoom and with the support of an educational platform in Canvas. However, the course has been redesigned to be carried out in an asynchronous modality, where the teacher fulfills a function of accompaniment and virtual advice. Likewise, the course has included gamification strategies where the student can obtain badges depending on their progress. In conclusion, it has been possible to demonstrate how information and communication technologies facilitate educational innovation for a correct design and implementation of virtual learning environments.
\end{abstract}

Palabras clave-- asynchronous, e-learning, research, TICs

Digital Object Identifier (DOI):

http://dx.doi.org/10.18687/LEIRD2021.1.1.20

ISSN: 2414-6390 ISBN: 978-958-52071-9-6 


\title{
Innovación educativa a través de tecnologías de la información y comunicación: estudio de caso en un curso de investigación formativa
}

\author{
Cardoza-Sernaqué Manuel Antonio, Maestro en gestión del talento humano ${ }^{1}$, Miñan-Olivos Guillermo Segundo, Magíster en \\ gestión pública ${ }^{1}$, Pulido-Joo Luis Alexander, Maestro en gestión del talento humano ${ }^{1}$, Dios Castillo, Christian Abraham, Magíster \\ en administración de negocios y Pelaez-Valdivieso José Victor, Doctor en Administración y Manrrique Luperdi Alexander \\ Miguel, Estudiante de Contabilidad ${ }^{1}$ \\ 1'Universidad Tecnológica del Perú, Perú, mcardoza@utp.edu.pe, c20342@utp.edu.pe, lpulido@utp.edu.pe, cdios@utp.edu.pe, \\ u21202726@utp.edu.pe \\ 2Universidad César Vallejo, Perú, mgjosepelaez@gmail.com
}

\begin{abstract}
Resumen- La presente investigación describe la innovación educativa a través de tecnologías de la información y comunicación y plantea un estudio de caso en un curso de investigación formativa donde la pandemia del Covid-19 conllevó la necesidad de replantear todo el proceso de enseñanza-aprendizaje. La investigación fue del tipo no experimental con un enfoque mixto (cualitativo y cuantitativo) y un alcance descriptivo. El curso analizado se dictaba en forma presencial y al inicio de la pandemia se convirtió en un curso virtual donde la clase se desarrollaba mediante Zoom y con el respaldo de una plataforma educativa en Canvas. Sin embargo, el curso ha sido rediseñado para que se realice en una modalidad asincrónica, donde el docente cumple una función de acompañamiento y asesoramiento virtual. Asimismo, el curso ha incluido estrategias de gamificación donde el estudiante puede ir obteniendo insignias en función de su avance. En conclusión, se ha podido demostrar como las tecnologías de la información y de la comunicación facilitan la innovación educativa para un correcto diseño e implementación de entornos de aprendizaje virtual.
\end{abstract}

Palabras clave-- asincrónico, e-learning, investigación, TICs

\section{INTRODUCCIÓN}

Las Tecnologías de la Información y la Comunicación (TICs) pueden apoyar la mejora de la calidad educativa, en la medida en cumplan algunas condiciones en su diseño, su implementación y su evaluación. Asimismo, las TICs, en la educación superior, representan novedosos entornos y oportunidades para el desarrollo de un aprendizaje a nivel individual y colaborativo [1].

En particular, las TICs pueden forzar y dar soporte a los necesarios cambios en las prácticas educativas, que permitan ajustarlas a las demandas de la sociedad del siglo XXI. Si no se toma en cuenta una incorporación estructurada, sistemática e integral de las tecnologías de información y comunicación, las organizaciones educativas no serán capaces de ajustar su comportamiento a características propias y personalizadas de sus estudiantes; lo que a su vez podría incurrir en deficiencias para su inserción laboral y en la sociedad en sí misma. Es por ello por lo que las tecnologías de la información y comunicación deben ser consideradas, en el campo de la educación, como un elemento fundamental y relevante para llevar a cabo innovaciones que generen un cambio significativo en el funcionamiento y desarrollo del aprendizaje [2]. Considérese innovación como el proceso que se presenta mediante una nueva forma de hacer las cosas reemplazando algún método tradicional en una comunidad y en el caso de la innovación educativa, se puede establecer como el proceso capaz de identificar restricciones y falencias en una matriz educativa tradicional para alterarla en beneficios de los estudiantes a través de novedosas estrategias pedagógicas [3]. Y si se llega a un nivel más profundo se estaría hablando de una innovación disruptiva, es decir, una innovación totalmente radical a través de un nuevo servicio o producto [4].

Justamente, muchas de las entidades educativas no estaban preparadas para un educación totalmente a distancia en el contexto del Covid-19 [5] lo cual conllevó a que muchas entidades educativas deban dar un giro transcendental en sus procesos formativos; sin embargo, dichos cambios no serán temporales ya que la educación virtual seguirá teniendo un rol fundamental incluso después de haber superado los estragos de la pandemia. El desarrollo tecnológico y la acelerada producción de conocimientos en el presente siglo constituyen argumentos que avalan la virtualidad como una revolución dentro de la educación, puesto que, desde finales del siglo XX, algunos especialistas vislumbraban que más temprano que tarde se produciría una transformación digital de los procesos educativos. Es cierto que la educación a distancia puede evidenciar una historia llena de diversas y sólidas experiencias pedagógicas, pero la actual pandemia ejerció un efecto catalizador para la migración de la tradicional modalidad presencial a una innovadora modalidad virtual [6].

La educación virtual significa otro lugar en el aula; un proceso educativo en el ciberespacio, una actividad de comunicación con intención de capacitación. Es una estrategia educativa que facilita el procesamiento de la información y permite la aplicación de nuevos métodos de enseñanza, que promueve el desarrollo de un aprendizaje significativo que se centra en los estudiantes y en su participación. El método de sincronización es aquel donde el que el emisor (docente) y el receptor (alumno) de un mensaje 
operan dentro del mismo período de tiempo durante la comunicación, es decir, deben aparecer dos personas al mismo momento para transmitir el mensaje. Estos recursos sincronizados son realmente necesarios para las actividades sociales, lo cual es indispensable, por lo tanto, los estudiantes que participan en modo virtual no se desenvolverán solos. A través de los métodos asincrónicos se pueden enviar mensajes sin hacer coincidir el remitente $y$ el receptor en una interacción instantánea. Requiere necesariamente una ubicación física y lógica, las almacena aquí y también puede acceder a los datos que componen el mensaje. Debido a que los estudiantes que aprenden en el modo de enseñanza virtual muestran características especiales, es absolutamente necesario acceder a la información en forma diferida, por lo que su uso en el modo de educación a distancia es más valioso [7].

\section{METODOLOGÍA}

El presente estudio ha tenido un enfoque mixto (cuantitativo y cualitativo), un diseño no experimental transversal y alcance descriptivo. Se tomó como estudio de caso, un curso de investigación formativa, que inicialmente se dictaba de manera sincrónica al inicio de la pandemia por Covid-19 y que, posteriormente, migró a una modalidad asincrónica. Durante el año 2021 se llevaron distintos cursos en modalidad asincrónica (población), pero tal como se ha mencionado, solo se tomó como muestra un curso, que fue seleccionado aplicando un muestreo no probabilístico por conveniencia. El procedimiento de investigación consistió en la descripción de los siguientes aspectos: diseño del curso asincrónico, analíticas web, acompañamiento virtual y gamificación.

\section{RESULTADOS Y DISCUSIÓN}

\section{Diseño del curso asincrónico}

El diseño del curso incluyó todo el material didáctico necesario para su desarrollo, es decir, se planteó un diseño sistematizado, planificado y estandarizado para que el curso se ejecute con estándares de calidad reproducibles en distintos contextos o realidades. Dicho criterio se tomó en cuenta ya que una de las principales dificultades de los docentes en cursos asincrónicos se relaciona con la elaboración de material didáctico y con el uso de herramientas tecnológicas [8]. Entre el material de estudio disponible, la inclusión de videos ha sido fundamental desde un punto de vista motivacional, instructivo y académico; sobre todo si se parte desde un enfoque del conectivismo, donde se incluye al estudiante por medio de canales educativos que no solo se limitan a la lectura, sino que, por el contrario, hacen uso de herramientas caracterizadas por aspectos de color y dinamismo, que resultan bastantes familiares para los estudiantes de la actualidad [9]. Por otro lado, el material audiovisual también buscó mantener una conexión con una figura que represente al docente tradicional pero dentro del ambiente de aprendizaje virtual.

A partir de la estrategia mencionada, el docente tutor se podía concentrar en realizar un acompañamiento personalizado del aprendizaje y convertirse en un aliado del estudiante para el cumplimiento del logro general de aprendizaje del curso. De la misma manera, el docente debía garantizar que el estudiante cumpla con lo establecido en el cronograma de actividades académicas del curso y para ello se ejecutaba un monitoreo constante. Por otro lado, el docente cumplía una función preventiva respecto a la entrega de trabajos calificados, asegurándose de una correcta difusión en las fechas o plazos de entrega correspondientes.

Las herramientas del curso estuvieron conformadas por material de lectura, material audiovisual, foros de debate, autoevaluaciones y prácticas. El curso presentaba una duración de 17 semanas y durante cada un de ellas se debían ejecutar actividades, las cuales fueron clasificadas como calificadas y no calificadas. La Tabla I muestra las actividades no calificadas individuales:

TABLA I

ACTIVIDADES INDIVIDUALES NO CALIFICADAS INCLUIDAS EN EL CURSO ASINCRÓNICO DE INVESTIGACIÓN

\begin{tabular}{|c|c|}
\hline Semanas & Título de la actividad \\
\hline 1 & $\begin{array}{l}\text { Autoevaluación - El plagio } \\
\text { Tarea - Practica el parafraseo } \\
\text { Autoevaluación - Productos de la investigación bibliográfica }\end{array}$ \\
\hline 2 & $\begin{array}{l}\text { Autoevaluación - El tema de investigación } \\
\text { Tarea - Practica sobre el tema de investigación } \\
\text { Autoevaluación - El problema de investigación } \\
\text { Practica sobre el problema de investigación }\end{array}$ \\
\hline 4 & Tarea - Búsqueda de fuentes de información \\
\hline 6 & Autoevaluación - Reporte de fuentes bibliográficas \\
\hline 7 & $\begin{array}{l}\text { Autoevaluación - Hipótesis } \\
\text { Autoevaluación - Esquema de investigación }\end{array}$ \\
\hline 9 & $\begin{array}{l}\text { Autoevaluación - Fichas textuales } \\
\text { Autoevaluación - Fichas de resumen } \\
\text { Tarea - Fichas textuales y de resumen }\end{array}$ \\
\hline 10 & $\begin{array}{l}\text { Ejercicio interactivo - Practica con los esquemas de redacción - } \\
\text { Parte } 1 \\
\text { Ejercicio - Practica con los esquemas de redacción - Parte } 2 \\
\text { Autoevaluación - Esquemas de redacción }\end{array}$ \\
\hline 12 & $\begin{array}{l}\text { Autoevaluación - Manejo de la información } \\
\text { Foro de debate - "El café" }\end{array}$ \\
\hline 15 & $\begin{array}{l}\text { Autoevaluación - Introducción } \\
\text { Autoevaluación - Conclusión }\end{array}$ \\
\hline
\end{tabular}

Fuente: Elaboración propia 
Las actividades no calificadas tenían por finalidad el fortalecimiento del aprendizaje antes de que los estudiantes procedan a desarrollar las actividades calificadas.

Por otro lado, el curso contempla la entrega de un producto final grupal que consiste en la presentación de un artículo académico. En ese sentido, en determinadas semanas se debe presentar un avance del producto, el cual tiene una calificación. En la Tabla II se muestran las actividades grupales que se califican y forman parte de la obtención del promedio general del curso.

TABLA II

ACTIVIDADES GRUPALES CALIFICADAS INCLUIDAS EN EL CURSO ASINCRÓNICO DE INVESTIGACIÓN

\begin{tabular}{|l|l|}
\hline Semanas & \multicolumn{1}{|c|}{ Título de la actividad } \\
\hline 3 & Asesoría 1 - Tema y problema de investigación \\
\hline 5 & Asesoría 2 - Fuentes de investigación \\
\hline 7 & Tarea Académica 1 \\
\hline 8 & Asesoría 3 - Hipótesis y esquemas de investigación \\
\hline 11 & Asesoría 4 - Esquema de redacción \\
\hline 12 & Tarea Académica 2 \\
\hline 15 & Asesoría 5 - Esquema de redacción (cuerpo) \\
\hline 16 & Asesoría 6 - Esquema de redacción (Introducción y conclusión) \\
\hline 17 & Trabajo final \\
\hline
\end{tabular}

Fuente: Elaboración propia

Para la asesoría 1, el grupo debía presentar adecuadamente un tema y problema de investigación, en ese sentido, la rúbrica de evaluación estipulaba como estándar esperado, una delimitación temática, espacial y temporal. Asimismo, se hacía necesario una correcta explicación del interés del grupo por el tema propuesto y una justificación que permita resaltar la importancia y el aporte del tema planteado.

Una vez planteado el tema y problema de investigación, el grupo debía presentar la asesoría 2. En esta actividad calificada, la presentación contenía las fuentes de investigación y la rúbrica de evaluación establecía como estándar esperado una correcta identificación bibliográfica de las fuentes, es decir, que el estudiante logre especificar aspectos como autor, tipo de publicación, institución de procedencia, entre otros, De la misma manera, se esperaba que el estudiante logre asegurar la pertinencia y confiabilidad de cada una de las fuentes, estableciendo el rigor científico y académico de cada una de ellas. Posteriormente a la asesoría 2 , se debía presentar la tarea académica 1 donde se recopilaban los avances previos con los mismos criterios de evaluación.

El caso de la asesoría 3, se trabajaba la hipótesis de investigación y un esquema de ideas acorde a la misma. En concordancia con ello, la rúbrica de evaluación establecía que la hipótesis debía responder adecuadamente al problema de investigación. Asimismo, se verificaba que cumpla con ciertos criterios como su condición aseverativa y directa. Cabe destacar, que en esta entrega se volvía a calificar tanto el tema como el problema de investigación, lo cual permitía que los grupos con mayor dificultad en el trabajo pudieran seguir corrigiendo esa parte del artículo.

En la asesoría 4, la presentación del trabajo grupal debía incluir todos los aspectos de la introducción del artículo y, que previamente ya se habían desarrollado: el tema, el problema, la justificación, la hipótesis, entre otros. Por otro lado, también se realizaba una presentación de las fuentes bibliográficas, ordenadas según los argumentos propuestos para comprobar la hipótesis de investigación. Y como último punto, se incluía un breve avance de la conclusión. Posteriormente, para la tarea académica 2 se presentaba todo el avance hasta la fecha, pero en la rúbrica de evaluación, se adicionaban criterios relacionados a la redacción de referencias bibliográficas utilizando las normas APA y al cumplimiento de aspectos ortográficos y de forma (tipo de letra, márgenes, etc.)

A partir de la asesoría 5, se iniciaba la revisión correspondiente a la redacción del cuerpo del artículo, es decir, a partir de la información recopilada en las fichas bibliográficas se planteaba la formación de párrafos con sus respectivos antecedentes y consecuentes; con la finalidad de que el estudiante pueda interpretar la información recopilada adecuadamente. En tal sentido, la rúbrica de evaluación estipulaba un correcto citado de la información mediante el uso de las normas APA, una coherencia en la presentación de la información, una adecuada síntesis de las ideas expuestas en cada argumento y el uso correcto de la puntuación y ortografía.

En el caso de la asesoría 6, se evaluaba los mismos criterios que en la asesoría 5, pero aplicados para las secciones de la introducción y conclusión. Para el trabajo final del curso, se debía presentar el artículo académico redactado de forma grupal. De esta manera, se lograba aplicar cada uno de los aspectos abordados semana a semana.

\section{Analíticas y evaluación del curso asincrónico}

Para una adecuada gestión del proceso de aprendizaje, el curso disponía de distintas analíticas para evaluar el avance y cumplimiento de los estudiantes. En la Figura 1 se puede observar el nivel de participación e interacciones por cada semana. 


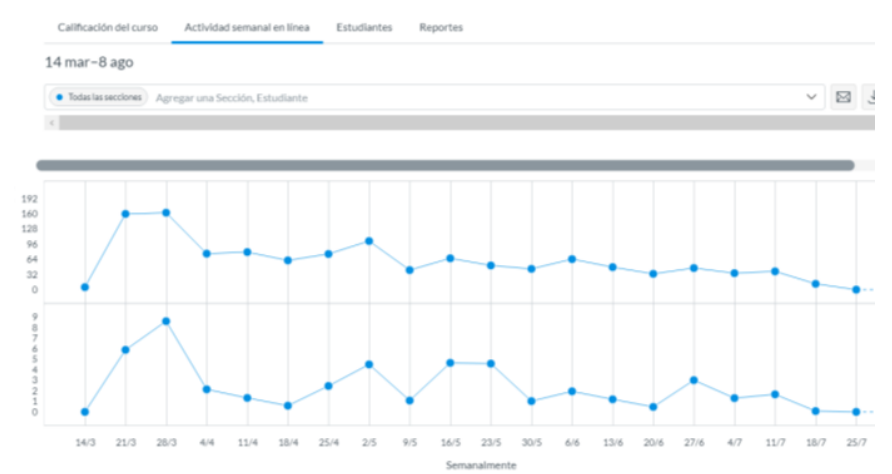

Fig. 1 Seguimiento semanal al cumplimiento de las actividades calificadas y no calificadas en curso asincrónico

Fuente: Elaboración propia

En la Figura 2 se puede observar el análisis que se podía realizar a cada uno de los estudiantes matriculados en el curso. Esta herramienta permitía visualizar calificaciones, porcentaje de tiempo, fecha de la última participación, fecha de la última visita al portal del curso, número de visitas y número de participaciones.

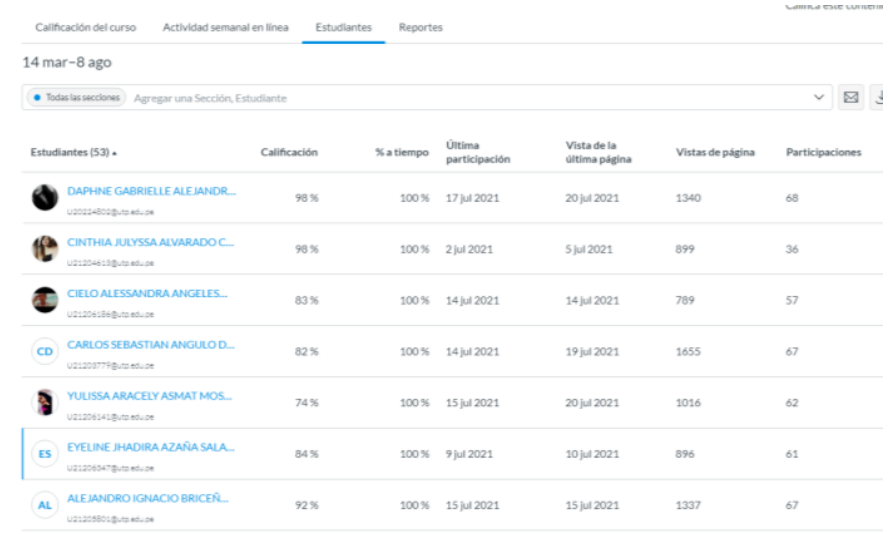

Fig. 2 Seguimiento a cada estudiante respecto a sus niveles de participación en el curso asincrónico

Fuente: Elaboración propia

Las interacciones de los estudiantes con el material educativo se reflejan en el número de visitas y de las participaciones. Un estudio sobre herramientas colaborativas asincrónicas incluyó el número de participaciones de los estudiantes en el uso de un blog educativo. Los resultados demostraron un total de 1000 comentarios registrados por cada semestre y una media semestral de 15 comentarios por cada estudiante [10]. En el caso del presente estudio, debido a que se trata de una herramienta más completa, el número de interacciones fue muy superior ya que se registró entre 800 y 1400 visitas a las páginas por cada estudiante, mientras que la participación media estuvo entre 30 y 70.

Las horas de conexión representaron un indicador clave dentro del curso asincrónico. Diversos autores mencionan que, en este tipo de cursos, la gestión del tiempo y la planificación del estudio resultan de vital importancia para el desenvolvimiento del estudiante y su respectivo rendimiento académico [11]. Asimismo, en la modalidad asincrónica se espera que cada estudiante tenga la habilidad de autorregular su propio aprendizaje [12]; es decir, cada uno de ellos debe ser consciente de su propio proceso cognitivo, socioafectivo y motivacional y, en ese sentido, existen diversas líneas teóricas que tienen un respaldo empírico.

Por otro lado, se debe tener en cuenta que la evaluación es un factor constitutivo en todo proceso de enseñanza aprendizaje, y por tal motivo, todo curso académico debe contar con el sistema necesario para reconocer los aciertos y dificultades de cada estudiante y que a su vez se pueda implementar las estrategias de acción para retroalimentar el proceso [13]. Asimismo, un método de evaluación adecuado debe aplicarse de forma continua, sistemática e integral; por lo cual garantiza que el proceso cuenta con un instrumento para brindar el apoyo necesario a cada uno de los estudiantes [14]. En la Figura 3 se puede evidenciar que por cada actividad calificada se podía llevar a cabo un análisis cuantitativo para determinar si el rendimiento académico de los estudiantes fue el esperado. Las métricas disponibles fueron: calificación promedio, promedio más bajo, promedio más alto, entregas deficientes y entregas con atraso. El análisis de la información se podía resumir por semana y por cada estudiante.

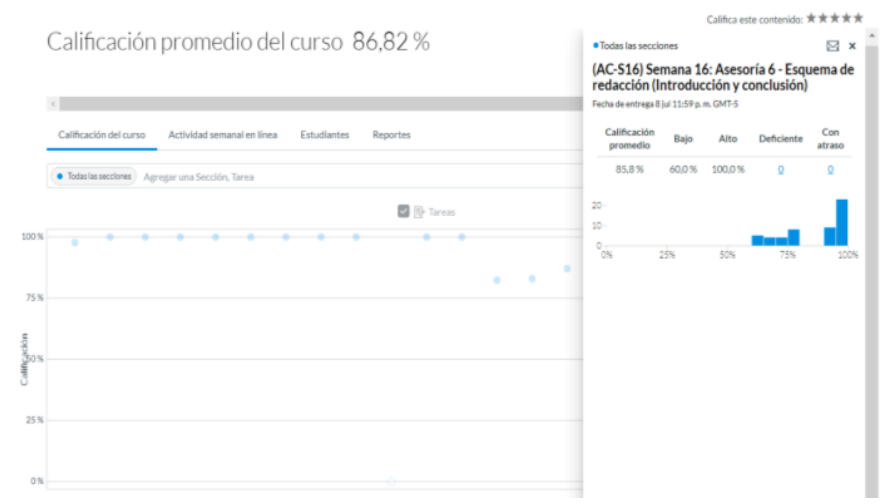

Fig. 3 Rendimiento académico por cada actividad calificada del curso asincrónico

Fuente: Elaboración propia

\section{Acompañamiento virtual del curso asincrónico}

Al no tener clases sincrónicas, el curso contaba con un docente de acompañamiento virtual encargado de guiar a los estudiantes durante todas las semanas. Es por ello, que la implementación de esta modalidad requirió la preparación de un grupo de docentes que migraron de las clases sincrónicas a los cursos asincrónicos. Al igual que en otros casos estudiados, las capacitaciones consideraron herramientas tales como reuniones sincrónicas, mesa de ayuda para el docente, capacitaciones del área especializada de la universidad, asesorías personalizadas, atención a consultas por correo electrónico [15]. Toda esta capacitación se enfocó en que el docente posea determinadas competencias en su rol como tutor virtual. Por otro lado, en que conozcan estrategias de 
tutoría virtual tales como: estrategias para la atención de dudas y reclamos, estrategias de animación y motivación, estrategias de seguimiento y estrategias de comunicación.

En cuanto a la estrategia relacionada con la atención de dudas y reclamos, se puso énfasis en la utilización de una comunicación escrita y oral totalmente clara, oportuna y amable; con la finalidad de que el estudiante pueda comprender las indicaciones y las respuestas dadas por el docente. En concordancia con lo mencionado, el docente no podía exceder las 24 horas para emitir una respuesta ante cualquier duda formulada por algún estudiante; la cual podía registrarse a través de distintos canales (bandeja de entrada, anuncios, foros, entre otros). Ante cada situación de cuestionamiento, el docente debía mantener una actitud positiva y asertiva para lo cual su control emocional era sumamente importante.

Con respecto a las estrategias de animación y motivación, se planteó la necesidad de que cada docente establezca las acciones necesarias para motivar constantemente a los estudiantes desde el inicio del semestre hasta el final del mismo. Es por ello que cada docente debía desarrollar espacios para poder presentarse y brindar la bienvenida a los estudiantes (anuncios y foros), al inicio del curso debía explicar la dinámica del curso y detallar el cronograma del mismo a través de una videoconferencia en Zoom, semanalmente debía iniciar con un anuncio informativo y motivador y, finalmente, debía evaluar la necesidad de algún mensaje motivador personalizado por bandeja de entrada (correo electrónico); sobre todo en aquellos estudiantes que presentaran algún dificultad.

Para las estrategias de seguimiento, cada docente debía de responder oportunamente a los trabajos presentados y a las consultas formuladas. Asimismo, el curso facilitaba herramientas para revisar el rendimiento académico de cada estudiante y así poder prestar atención a los estudiantes con mayores limitaciones en el desarrollo de las actividades calificadas. El docente debía asegurarse del cumplimiento de cada actividad y la meta propuesta como indicador era un cumplimiento del $70 \%$ de los estudiantes en actividades calificadas.

Para el caso de la estrategia de comunicación, los docentes fueron capacitados para emplear un estilo de comunicación asertivo y empático con cada uno de los estudiantes. Como recomendaciones específicas, se mencionó que los educadores debían dirigirse a los estudiantes de manera personal, es decir, por su nombre para establecer lazos de confianza. De la misma manera, se debía considerar las felicitaciones públicas cuando se detecten actitudes positivas en los estudiantes, mientras que, si era necesario una llamada de atención, esta sea de manera privada.
Cada una estas estrategias concuerdan con lo expuesto por otros autores. Por ejemplo, respecto al acompañamiento pedagógico en una asignatura virtual, un estudio midió la percepción de los estudiantes en los siguientes puntos: comunicación, organización de la asignatura, selección de contenidos y bibliografía, trabajos prácticos, instancias de valoración y resultados de aprendizaje [16].

En la figura 4 se puede observar los 3 ejes fundamentales para el curso asincrónico. En primer lugar, los foros de consulta para atender las inquietudes y dudas de los estudiantes. En segundo lugar, el feedback que se brindaba a cada una de las actividades calificadas y no calificadas para que el estudiante tenga la capacidad de corregir algunos errores o de fortalecer los aciertos obtenidos en cada una de las tareas. Finalmente, a pesar del diseño asincrónico del curso, se consideró necesario reuniones esporádicas en algunas semanas mediante la herramienta de Zoom para que los estudiantes puedan anticipar dudas de manera más directa.

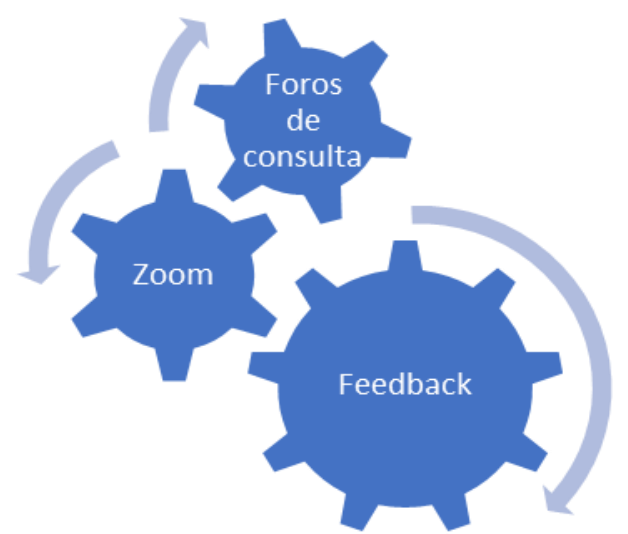

Fig. 4 Herramientas de acompañamiento virtual para el curso asincrónico Fuente: Elaboración propia

\section{Gamificación del curso asincrónico}

Los recursos de gamificación conforman una estrategia enfocada en la realización de juegos, en contextos no lúdicos, con la finalidad de que el docente tenga la capacidad de desarrollar un interés y aprendizaje significativo en los estudiantes [17].

En el caso de la presente investigación, la gamificación se utilizó como estrategia de motivación e incentivo para los estudiantes. Dependiendo de su avance, se le otorgaba una insignia. Por ejemplo, para los estudiantes que completaban satisfactoriamente la asesoría 1, durante la semana 3, se les otorgaba la insignia de "investigador novato", posteriormente, luego de haber completado la asesoría 2, en la semana 5 , se le entrega un reconocimiento como practicante de investigador. 


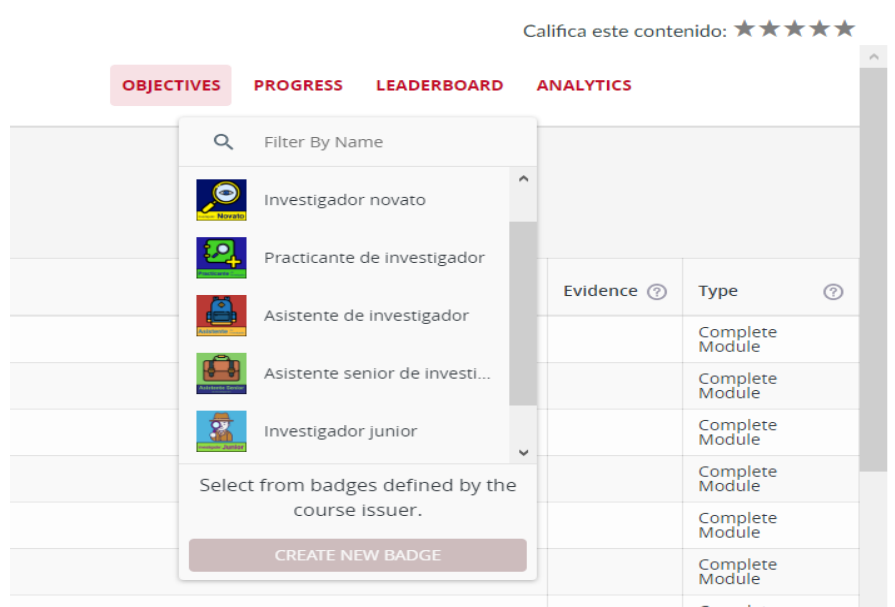

Fig. 4 Insignias otorgadas en el curso asincrónico según su nivel de avance en las actividades

Fuente: Elaboración propia

\section{CONCLUSIONES}

El presente estudio ha demostrado lo importante de las tecnologías de la información y comunicación para la innovación educativa en entornos de aprendizaje virtual. Dicha importancia se ha podido corroborar a través del estudio de un curso en modalidad asincrónica, donde se evidencia que el aprendizaje no presencial puede realizarse asegurándose estándares educativos de calidad. En dicho contexto, las tecnologías de la información y comunicación facultan a las instituciones de educación superior de herramientas digitales para que los estudiantes dispongan de toda la información académica necesaria, para que los docentes apliquen estrategias de seguimiento del aprendizaje y para que los sistemas de evaluación sean confiables en función al logro de competencias. Asimismo, los avances tecnológicos están permitiendo incluir nuevas estrategias como la gamificación en entornos de aprendizaje virtual.

\section{REFERENCIAS}

[1] Navarro, S., "Tendencias en el uso de recursos y herramientas de la tecnología educativa en la educación universitaria ante la pandemia COVID-19", Revista de Ciencia y Tecnología El Higo, vol. 10, no. 2, pp. 111-122, Diciembre 2020

[2] TICS en educación: una innovación disruptiva, Banco Interamericano de Desarrollo.

https://publications.iadb.org/publications/spanish/document/TICS-enEducación-Una-Innovación-Disruptiva.pdf

[3] Chávez, Z. y Martinez, H., "Gestión del conocimiento, creatividad e innovación en la educación universitaria venezolana", Negotium Revista Científica Electrónica de negocios, vol. 10, no. 2, pp. 5-17, Diciembre 2020

[4] Cabero, J., "Los MOOCS: de innovación disruptiva a nuevo modelo de negocio en educación superior", Educación y Comunicación, vol. 1, no. 9, pp. 27-39, Noviembre 2014

[5] Guiot, I., "Uso de las TICS en la educación superior durante la Pandemia COVID-19: Ventajas y desventajas", Interconectando Saberes, vol. 6, no. 12, pp. 223-227, Julio 2021

[6] Cayo, C. y Agramonte, R., "Desafíos de la educación virtual en Odontología en tiempos de pandemia COVID-19", Revista Cubana de Estomatología, vol. 16, no. 48, pp. 1-5, Julio 2020.

[7] Quintero, J., "El Efecto del COVID-19 en la Economía y la Educación: Estrategias para la Educación Virtual de Colombia", Instituto
Internacional de Investigación y Desarrollo Tecnológico Educativo INDTEC, vol. 57, no. 3, pp. 5-17, Julio 2020.

[8] Yanza, W., Montoya, J., Benítez, L. y Samaniego, A., Dificultades docentes en el uso de herramientas sincrónicas y asincrónicas en los docentes universitarios, Polo del conocimiento, vol. 5, no. 1, pp. 799-815, noviembre 2020

[9] Contreras, L., "Mejorando la experiencia del usuario en ambientes virtuales de aprendizaje SENA "English Dot Works", Rutas De formación: Prácticas y Experiencias, vol. 1, no. 8, pp. 128-134, enero 2019.

[10]Tobar, A., Lozada, R. y Maldonado, C., "Herramientas colaborativas asincrónicas en el proceso de enseñanza aprendizaje académico", Dominio de las ciencias, vol. 3, no. esp., pp. 439-453, marzo 2017.

[11]Zambrano, C., Bravo, I., Maluenda, J. y Infante, V., "Planificación y uso del tiempo académico asincrónico de estudiantes universitarios en condiciones de pandemia", Formación Universitaria, vol. 14, no. 4, pp. 113-122, agosto 2021.

[12]Delen, E., Liew, J. y Willson, V., "Effects of Interactivity and Instructional Scaffolding on Learning: Self-regulation in Online Videobased Environments", Computers \& Education, vol. 78, no. 1, pp. 312320, septiembre 2014.

[13]Lescano, L. y Vilanovo, G., "Reflexión sobre la educación en la formación universitaria", Revista Panamericana de Pedagogía, vol. 1, no. 5, pp. 113-130, enero 2004.

[14]Crosetti, V., Caggiano, C. y Casella, N., "La importancia de los recursos virtuales en épocas de pandemia. El curso de Química Analítica I de la UNNOBA como caso de estudio", Revista Iberoamericana de Tecnología en Educación y Educación en Tecnología, vol. 1, no. 28, pp. 83-92, abril 2021.

[15]Garmendía, A., Rainolter, M., Fuertes, M. y Senger, M., Acciones de acompañamiento de un equipo de la Unidad de Apoyo Central (UAC) del SIED para la continuidad académica en el contexto de emergencia, Sistema Internacional de Educación a Distancia, vol. 1, no. 3, pp. 7-18, mayo 2021.

[16]Del Valle, M., Salazar, N., Lara, C. y Villa, H., "Percepción de las y los estudiantes sobre el acompañamiento pedagógico virtual de la asignatura Metodología de la Investigación Clínica y Bioestadística Aplicada en la Carrera de Medicina de marzo a julio 2020", Revista Argentina de Educación Médica, vol. 9, no. 4, pp. 7-13, diciembre 2020.

[17]Zambrano, S. y Marcillo, C., "La gamificación como estrategia de comunicación asincrónica en la educación Básica Superior", Dominio de las ciencias, vol. 7, no. 1, pp. 971-986, enero 2021.

$1^{\text {sh }}$ LACCEI International Multiconference on Entrepreneurship, Innovation and Regional Development - LEIRD 2021: "Ideas to Overcome and Emerge from the Pandemic Crisis", Virtual Edition, December 9-10, 2021. 